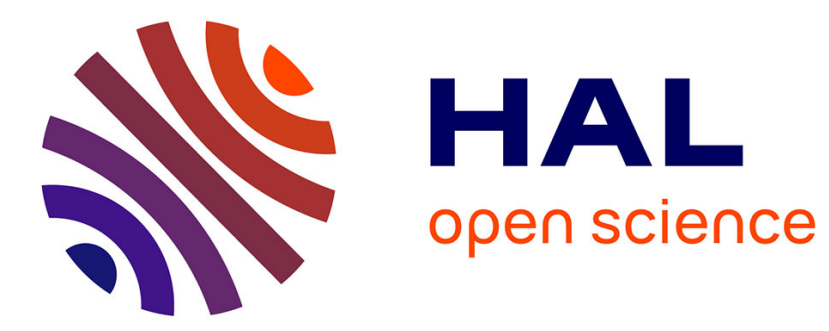

\title{
Les trajectoires de l'eau et de la terre dans la construction des territoires
}

\author{
Julie Trottier
}

\section{To cite this version:}

Julie Trottier. Les trajectoires de l'eau et de la terre dans la construction des territoires. Espace Géographique, 2015, 2, pp.97-102. 10.3917/eg.442.0097 . hal-02111549

\section{HAL Id: hal-02111549 \\ https://hal.science/hal-02111549}

Submitted on 26 Apr 2019

HAL is a multi-disciplinary open access archive for the deposit and dissemination of scientific research documents, whether they are published or not. The documents may come from teaching and research institutions in France or abroad, or from public or private research centers.
L'archive ouverte pluridisciplinaire HAL, est destinée au dépôt et à la diffusion de documents scientifiques de niveau recherche, publiés ou non, émanant des établissements d'enseignement et de recherche français ou étrangers, des laboratoires publics ou privés. 
Introduction du dossier soumis à l'Espace Géographique

\section{Titre: Les trajectoires de l'eau et de la terre dans la construction des territoires}

\section{Title: The trajectories of water and land in the construction of territories}

Auteur: TROTTIER, Julie, CNRS, Centre de Recherche Français à Jérusalem, 3 rue Shimshon, Jérusalem. Julie.trottier@cnrs.fr

Nombre de signes, incluant les espaces: 20624

L'étude des bouleversements de la gestion de la terre et de l'eau a tendance à suivre des effets de mode. Ainsi, la privatisation de l'eau et la question des grands barrages firent l'objet d'une littérature abondante dans les années 1990 et 2000. (Swyngedouw 1997; Bakker 2003; Page 2005; Castro 2007) Depuis 2009, une littérature très riche se développe sur l'accaparement de terres à grande échelle. (Chouquer and foncière 2012) (Borras, Fig et al. 2011) Ces sujets méritent évidemment l'intérêt qu'ils soulèvent. Mais l'attention massive qu'ils suscitent chez les chercheurs peut mener ces derniers à privilégier indument ce qui se révèle, à long terme, un épiphénomène et à négliger d'autres mécanismes au moins tout aussi importants. Ainsi, la vague d'investissements de la part des grandes compagnies d'eaux dans le monde en développement entama un reflux au début des années 2000. Et si les investissments fonciers à grande échelle bouleversent aujourd'hui la planète, d'autres mécanismes comme l'agriculture sous contrat se répandent au moins aussi rapidement. Braquer les projecteurs sur les grands investissements de compagnies issues de pays riches nous permet surtout de suivre la logique et les stratégies de ces acteurs. Une autre approche est nécessaire si nous souhaitons comprendre la façon dont le rapport à la terre et à l'eau se transforme aujourd'hui et change par conséquent la façon complexe dont diverses catégories d'acteurs s'approprient l'espace et les ressources. Par ailleurs, la littérature sur le foncier et celle sur l'eau se sont rarement nourries l'une de l'autre. Il importe d'intégrer les apports théoriques de l'une et de l'autre pour comprendre la façon dont la transformation multi-échelle des rapports à l'eau et à la terre participe aujourd'hui à la construction des territoires. 
Le projet "De terres et d'eaux" regroupa à partir de 2013 des géographes, politologues, agronomes, ethnographes et cartographes pour explorer de façon concomitante la trajectoire de l'eau au travers des diverses formes d'organisations sociales, formelles et informelles, qui régissent sa gestion et celle de la terre en tant qu'objet idéel et matériel de systèmes de tenure en constante évolution. Ce dossier est issu de ce projet collectif. Il utilise six études de cas locales, menées au Kenya, en Ouganda, en Territoires Palestiniens, au Teraï, en Espagne et au Mozambique pour examiner les trajectoires de la terre et de l'eau, ainsi que les processus de construction territoriale qu'elles engendrent aujourd'hui sur des échelles allant du très local au global.

Aujourd'hui, la transformation globale de la production agricole entraîne tout aussi bien un bouleversement des constructions sociales régissant les rapports à la terre que de celles régissant les rapports à l'eau. Il importe de comprendre les processus de construction des territoires en cours au travers de ces transformations. La littérature sur les acquisitions massives de terre a initialement abordé cette transformation du point de vue du foncier et des investissements à grande échelle. Elle nous offre aujourd'hui un nombre croissant d'études de cas locaux permettant de mieux appréhender l'impact d'un phénomène global sur une échelle locale. Les études sur l'eau d'une part et sur le foncier d'autre part ont jusqu'à présent surtout adopté une approche déductive. Elles montrent souvent un cadre théorique bien développé, ancré dans une political ecology empreinte d'une approche marxiste ou, au contraire, dans un environnementalisme empreint d'une approche néolibérale. Les littératures sur la privatisation de l'eau et sur le land grab illustrent largement le premier cas et celle sur les paiements des services écosystémiques, le second cas.

On doit se poser la question du choix de ces approches. Pourquoi les chercheurs ont-ils privilégié l'étude des investisseurs étrangers, que ce soit dans la terre ou dans l'eau? Pourquoi les chercheurs ont-ils adopté des méthodes déductives à partir de théories très élaborées, qu'elles soient ancrées dans le marxime ou le néolibéralisme? Il est plus aisé pour un chercheur occidental d'enquêter sur un investisseur globalisé que sur une communauté agricole isolée et dépourvue d'électricité. Le premier rédige ses 
contrats dans une langue lue par le chercheur. Ses avocats ont étudié le même droit étatique que le chercheur et ses pratiques quotidiennes, comme la prise de rendez-vous, se prêtent bien à des interviews programmées lors d'un travail de terrain court. Par contre, enquêter auprès de communautés agricoles isolées, vivant sans électricité, requiert un travail de terrain beaucoup plus long dont peu de chercheurs disposent. Essentiellement basés dans le monde industrialisés, les chercheurs privilégient d'ordinaire une approche déductive, au sein de laquelle leur cadre théorique trés élaboré se complète d'enquêtes de terrain limitées. Les conditions de travail mêmes des chercheurs ont ainsi créé un biais méthodologique dans leur approche de la terre et de l'eau.

Notre collectif a développé une approche inductive, qui explore les interactions entre les acteurs impliqués dans la gestion de l'eau et de la terre, en prenant comme point de départ leur interaction avec la ressource. Cette approche inductive exclut une identification préalable des acteurs importants. Notre travail au Mozambique révéla ainsi une absence des investisseurs étrangers là où leur propres publications les proclament présents. Il identifia en revanche nombre d'acteurs locaux importants, comme les microcultivateurs de cotton, impliqués dans l'agriculture sous contrat. Notre travail en Palestine permit de distinguer les interactions entre propriétaires fonciers palestiniens absents, leurs métayers, propriétaires palestiniens exploitants et colons israéliens dans une mosaïque territoriale complexe. Cette approche inductive exclut une identification préalable des enjeux et des processus les plus importants. Notre travail en Espagne révéla le rôle de choix à court terme comme, par exemple, celui de cultiver du riz pour nourrir les troupes franquistes pendant la guerre civile espagnole. Il révéla aussi l'importance de la navigation entre le golfe de Cadix et Séville dans la construction du territoire des marais. Les travaux hydrauliques firent progresser l'eau salée à l'intérieur des terres et isolèrent les terres de Doñana des rivières qui alimentaient autrefois ses marais. En résulte aujourd'hui une alliance entre environnementalistes défendant le parc national de Doñana et riziculteurs. Par contre, le travail réalisé au Kenya explore l'échec de l'alliance préconisée entre "utilisateurs (acheteurs) des services écosystémiques", les producteurs industriels de roses de l'aval du bassin du lac Naivasha et "fournisseurs (vendeurs)" de ces services, les paysans kikuyu qui, en adoptant des mesures anti-érosion, empêcheraient la sédimentation du lac. 
En permettant de s'émanciper des hypothèses sous-jacentes aux approches déductives, notre approche révèle l'importance d'acteurs et d'enjeux souvent négligés sinon ignorés. Ce choix méthodologique a entraîné deux difficultés corrélatives: la longueur des terrains, d'une part, et la complexité des résultats à théoriser d'autre part. Notre collectif a réalisé des travaux de terrain longs - plus de deux ans en Ouganda, en Palestine et au Terai - qui lui ont permis de reconstituer les territoires vécus par les acteurs en fonction de leurs rapports à l'eau et à la terre et ce qu'ils en font. Ce travail a mis en évidence les liens fonctionnels qui relient des espaces à priori dispersés. Le projet a réalisé des travaux de terrain dans des zones particulièrement difficiles comme des régions agricoles dépourvues d'électricité au Mozambique et des régions du Liban dont le niveau de sécurité n'a cessé de fluctuer - permettant d'identifier des acteurs souvent négligés par la recherche et d'explorer leurs stratégies. Il a aussi exploré des régions particulièrement bien intégrées dans l'économie globalisée, qu'il s'agisse des marais du Guadalquivir en Espagne, ou du lac Naivasha au Kenya. Ces deux derniers cas ont mis en évidence le processus d'hybridation des discours environnementalistes avec les réalités locales de construction territoriale.

En suivant ainsi "d'en bas" les processus de construction territoriale par rapport à l'eau et à la terre, notre collectif a pu mettre en lumière le rôle des acteurs locaux par rapport à ceux, beaucoup plus étudiés, des investisseurs internationaux et des Etats. Rôle à la fois dans la construction discursive de ce qu'est la nature, ce qui constitue un bon choix technologique, ce qu'il est légitime de s'approprier et de ne pas s'approprier et dans la construction des règles à respecter. En Ouganda, la politique environnementaliste promue dans les années 1990 et 2000, cède aujourd'hui la place à une politique productiviste. En Espagne, par contre, une politique productiviste adopte des énoncés environnementalistes sans pour autant modifier ses pratiques en profondeur. Dans les deux cas, des acteurs locaux non étatiques ont joué un rôle majeur pour aboutir à cette transformation de la politique étatique.

Tous nos cas d'étude ont révélé des situations de pluralisme juridique auquel les acteurs extérieurs doivent s'adapter sous peine d'échouer dans leurs projets. Le cas Ougandais nous montre ainsi que les terres du marais d'Olweny appartiennent à l'Etat ougandais selon la loi nationale. Pourtant, les ayant droit du projet de riziculture, qui 
n'est même pas encore fonctionnel, se sont d'ores et déjà attribués un droit d'usage transférable par succession patrilinéaire, ce qui leur permet d'effectuer des souslocations impossibles en droit positif ougandais. Similairement, le cas palestinien révèle des "rentiers de l'eau" qui n'ont jamais habité le village où ils détiennent des parts d'eau qu'ils louent 400 dinars jordaniens par an. Ces réalités de terrain ne sont pas anecdotiques. L'appropriation de la terre et de l'eau s'effectue par des règles souvent énoncées localement. Différentes sources de droit seront invoquées par les mêmes acteurs en fonction des arènes où ils se trouvent. Le pluralisme juridique a déjà été étudié d'un point de vue anthropologique ou juridique. (Meinzen-Dick and Pradhan 2001) (Boelens 2009) Il importe d'incorporer ce pluralisme juridique dans notre théorisation de la construction territoriale. En effet, un aspect du "piège territorial" consiste à projeter une homogénéité juridique au sein d'un territoire.

Ce travail illumine la rencontre entre économies humaines d'une part et économies commerciales d'autre part. Les premières sont des systèmes économiques essentiellement soucieux de créer, détruire et redisposer des êtres humains plutôt que d'accumuler des richesses. (Graeber 2013) (p.160)Dans ces économies, ne peut être vendable que ce qui est d'abord arraché à son contexte. Graeber accorde donc beaucoup d'attention au "processus qui déloge les personnes des réseaux d'engagement mutuel, d'histoire commune et de responsabilité collective qui font d'eux ce qu'ils sont, pour les rendre échangeables - c'est-à-dire susceptible d'être assujettis à la logique de la dette." (Graeber, 2013, p.201) La violence de cette rencontre entre économies humaines et économies commerciales, qui aboutit à l'asservissement pour dettes des membres des premières par les membres des secondes, a motivé les recherches sur la privatisation de l'eau et sur les accaparements de terre. Une partie importante de cette violence repose dans la façon dont de nouvelles infrastructures affectent les modalités d'accès à l'eau des uns et des autres, lorsque des acteurs insérés dans une économie commerciale, aujourd'hui globalisée, interagissent avec des acteurs insérés dans une économie humaine. Elle repose aussi dans la façon dont le pluralisme juridique concernant la tenure foncière et l'eau sera maintenu ou transformé par les uns et les autres. Il importe de comprendre la façon dont l'évolution du rapport à l'eau et à la terre participe au processus d'assujettissement à la dette. Autrement dit, il nous faut comprendre la façon dont la construction discursive concernant la "bonne" gestion de l'environnement peut 
dépouiller les utilisateurs existants de la terre et de l'eau de toute légitimité dans ce rôle. Il nous faut comprendre aussi la façon dont ces acteurs réagissent en s'appropriant souvent en retour les discours environnementalistes, économiques et technologiques.

Le territoire comme méthode

Il importe enfin de discuter pourquoi notre collectif a recouru à l'approche territoriale pour son étude et la signification qu'il a donné à ce terme polysémique. Nous avons suivi Elden dans sa conceptualisation d'un territoire en tant que "technologie politique comprenant des techniques pour mesurer la terre et contrôler le terrain". (Elden 2010) (p.811)Les bouleversements que nous étudions se réalisent cependant essentiellement en dehors du monde occidental et nous cherchons à comprendre le rôle joué par les acteurs locaux, majoritairement non occidentaux. Elden a focalisé son travail sur les notions de territoire en Occident. En résulte une focalisation sur l'Etat comme seul acteur produisant le droit et comme seule source de violence organisant le bornage d'un espace qu'il contrôle. Ainsi Elden n'a pas intégré, par exemple, la notion d'assabiya de Ibn Khaldun qui définit la solidarité au sein d'une collectivité humaine sans la lier au maintien de l'ordre à l'intérieur d'un bornage territorial fixe et un espace délimité. (Khaldun 1997) Elden n'a pas intégré le pluralisme juridique qui est dominant dans le monde non-occidental. Ici, plusieurs sources de pouvoir exercent le contrôle social sur la terre et l'eau. Le modèle du "state-in-society" prédomine. (Migdal 2001) Nos cas d'étude illustrent la façon dont le pluralisme juridique participe à la construction de territoires emboîtés les uns dans les autres, souvent sans grand égard pour le territoire national.

Elden retient la notion de terre (land) comme liée à la propriété, comme une marchandise qui peut faire l'objet d'échanges parfaits tels qu'ils sont généralement conçus en occident. Notre approche interdisciplinaire a permis d'intégrer l'apport de la littérature sur le foncier, qui désigne les rapports qu'entretiennent les hommes entre eux au sujet de leur relation à la terre. La tenure foncière dans le monde non occidental ne se résume pas à la simple propriété. Sa complexité repose notamment sur les échanges imparfaits qui voient des relations de long terme naître entre les acteurs en raison d'une transaction foncière, relations qui s'avèrent de véritables pierres angulaires de la construction territoriale. (Le Roy, Karsenty et al. 1996) Elden a mis en avant la 
violence inhérente des techniques de relevés topographiques qui ont contribué au succès des Etats, en tant que formes d'organisation sociale capable de s'approprier le monopole du contrôle sur un espace donné. Si ce monopole n'a jamais été atteint dans la plupart des Etats issus de la décolonisation, le rôle politique des sciences et techniques n'en est pas moindre. L'étude des sciences et sociétés a théorisé la co-production de l'ordre naturel et de l'ordre social. (Jasanoff 2004) Lorsque la science change notre perception de la nature, elle change inéluctablement notre société. Conversément, la manière dont nous voulons gérer notre environnement est indissociable de la façon dont nous produisons nos énoncés scientifiques pour le comprendre. Notre étude a exploré les discours scientifiques et les choix technologiques comme le dragage d'une rivière ou la modélisation de sa salinité, comme participant à la co-production de l'ordre naturel et de l'ordre social. Ceci fait partie intégrante de la construction du territoire.

Des territoires multisitués aux territoires polytopiques

Notre collectif a laissé l'unité de bornage du territoire émerger du travail d'observation selon la méthode du grounded theory (Glaser and Strauss 1967). Nous n'avons considéré l'Etat que comme un acteur parmi d'autres. Tout acteur qui, par ses stratégies discursives ou matérielles s'approprie une ressource ou un espace participe selon nous à la construction d'un territoire. Notre perspective "d'en bas" a privilégié l'étude des acteurs directement en rapport avec la terre et l'eau et a inclus l'Etat parmi les autres. Récemment, la géographie a insisté sur la tri-dimensionalité des territoires (Weizman 2007) (Elden 2013). En utilisant la notion de territoires multisitués nous avons conceptualisé des territoires de plus de trois dimensions à la manière des polytopes de Boole. Les territoires multisitués peuvent être compris comme un ensemble de dispositifs spatiaux dont les entités sont dispersées dans l'espace mais reliées fonctionnellement. (Giraut 2013) En nous focalisant sur les rapports discursifs et matériels entretenus par les acteurs avec l'eau et la terre, nous avons laissé l'unité de bornage émerger en fonction du territoire vécu par les acteurs. Les liens fonctionnels entre les espaces appropriés nous ont fourni les dimensions selon lesquelles on pouvait les agencer en polytopes à plus de trois dimensions. De tels polytopes sont perçus, au sein d'un monde en trois dimensions, comme une série de de formes tridimensionnelles déconnectées les unes des autres alors qu'elles ne forment en réalité qu'un seul objet en 
quatre dimensions, ou plus. Ceci fait notamment apparaître dans le cas palestinien, les liens fonctionnels entre de nombreux espaces a priori dispersés qui forment collectivement un seul territoire polytopique imbriqué dans d'autres territoires polytopiques. Plutôt que de considérer un territoire tri-dimensionel de la terre et de l'eau, nous plaidons plutôt pour un territoire polytopique de la terre et de l'eau qui nous apparaît à priori, dans une carte à deux dimensions ou dans une approche à trois dimensions, comme un territoire multisitué.

Bakker, K. J. (2003). An uncooperative commodity : privatizing water in England and Wales. Oxford: Oxford University Press.

Boelens, R. (2009). "The Politics of Disciplining Water Rights." Development and Change 40(2): 307-331.

Borras, S. M., D. Fig, et al. (2011). "The politics of agrofuels and mega-land and water deals: insights from the ProCana case, Mozambique." Review of African Political Economy 38(128): 215-234.

Castro, J. E. (2007). "Poverty and citizenship: Sociological perspectives on water services and public-private participation." Geoforum 38(5): 756-771.

Chouquer, G. and F. i. e. foncière (2012). Terres porteuses entre faim de terres et appétit d'espace. Arles: Actes Sud

Elden, S. (2010). "Land, terrain, territory." Progress in Human Geography 34(6): 799-817.

Elden, S. (2013). "Secure the volume: Vertical geopolitics and the depth of power." Political Geography 34: 35-51.

Giraut, F. (2013). "Territoire multisitué, complexité territoriale et postmodernité territoriale: des concepts opératoires pour rendre compte des territorialités contemporaines?" L'Espace géographique 42: 293-305.

Glaser, B. G. and A. L. Strauss (1967). The discovery of grounded theory. Chicago: Aldine.

Graeber, D. (2013). Dette 5000 ans d'histoire. Paris: Les Liens qui Libèrent.

Jasanoff, S. (2004). States of knowledge : the co-production of science and social order. London: Routledge.

Khaldun, I. (1997). Discours sur L'Histoire Universelle Al-Muqaddima. Arles: Actes Sud. 
Le Roy, É., A. Karsenty, et al. (1996). La sécurisation foncière en Afrique pour une gestion viable des ressources renouvelables. Paris: Karthala.

Meinzen-Dick, R. S. and R. Pradhan (2001). "Implications of legal pluralism for natural resource management." Ids Bulletin-Institute of Development Studies 32(4): 10-20.

Migdal, J. S. (2001). State in Society. Cambridge, Cambridge University Press.

Page, B. (2005). "Paying for water and the geography of commodities." Transactions of the Institute of British Geographers. 30(3): 293-306.

Swyngedouw (1997). Power, nature and the city. The conquest of water and the political ecology of urbanization in Gayaqiul, Ecuador: 1880-1990. Environment and Planning A, vol.29: 311-332.

Weizman, E. (2007). Hollow land : Israel's architecture of occupation. London, Verso. 